\title{
FAUNA DE COLEÓPTEROS SCARABAEOIDEA DE SANTO DOMINGO HUEHUETLÁN, PUEBLA, MÉXICO. SU POTENCIAL COMO INDICADORES ECOLÓGICOS
}

\author{
Gonzalo YANES-GóMEZ ${ }^{1}$ y Miguel-Angel MORóN² \\ 1Escuela de Biología, BUAP, Edif. 76, Ciudad Universitaria, CP 72570, Puebla, Puebla, MÉXICO. \\ E-mail: ggonzale@ siu.buap.mx \\ ${ }^{2}$ Red de Biodiversidad y Sistemática, Instituto de Ecología, A.C., Apartado Postal 63, \\ Xalapa, Veracruz 91000, MÉXICO. E-mail: miguel.moron@ inecol.edu.mx
}

Yanez-Gómez \& M. A. Morón. 2010. Fauna de coleópteros Scarabaeoidea de Santo Domingo Huehuetlán, Puebla, México. Su potencial como indicadores ecológicos. Acta Zoológica Mexicana (n.s.), 26(1): 123-145.

RESUMEN. En este trabajo se analizan la riqueza, diversidad, abundancia y las especies indicadoras de diversidad de coleópteros Scarabaeoidea recolectados en el bosque tropical caducifolio (BTC) y zona agrícola (ZA) ubicados en el sur del Municipio de Santo Domingo Huehuetlán, Puebla, durante los meses de octubre de 2005 a septiembre de 2006. Se encontraron 1,020 ejemplares adultos que representan a 52 especies incluidas en 29 géneros de tres familias de Scarabaeoidea, y 113 larvas edafícolas de 5 géneros, 6 especies y 9 morfoespecies de Melolonthidae. Se elaboraron curvas de acumulación de especies y se estimó la abundancia por sitio, por mes y por método de colecta. Se hizo un análisis comparativo entre coleópteros adultos del BTC y la ZA capturados con trampas, y se encontraron 34 y 36 especies, y 603 y 417 individuos en cada sitio respectivamente. El índice de diversidad de Shannon-Wiener fue de 2.79 para ZA y de 2.67 para BTC. Asimismo, se encontraron 82 larvas de cuatro géneros en BTC, y 30 larvas de cuatro géneros en ZA. Se emplearon adultos de 23 especies indicadoras en BTC y 21 en ZA, pertenecientes a las subfamilias Dynastinae, Rutelinae, y Scarabaeinae. La abundancia de especies indicadoras fue de 313 y 245 y el índice de diversidad fue de 2.24 y 2.43 en ZA y BTC respectivamente. Se concluye que la composición de especies de ambas comunidades es parecida (49\%), y que el efecto antrópico sobre el bosque como resultado de las actividades agrícolas aún es mínimo.

Palabras clave: Melolonthidae, Scarabaeidae, Trogidae, adultos, larvas, Ecología, bosque tropical caducifolio.

Yanez-Gómez \& M. A. Morón. 2010. The scarab beetles of Santo Domingo Huehuetlan, Puebla, Mexico. Their potential as ecological indicators. Acta Zoológica Mexicana (n.s.), 26(1): 123-145.

ABSTRACT. The richness, diversity, abundance and indicator species of diversity of adults and larvae of scarab beetles from Santo Domingo Huehuetlán, Puebla, México is analyzed. The field work was done between October 2005 and September 2006, obtaining 1,020 adults specimens of 52 species included in 29 genera from three families of Scarabaeoidea, and 113 edaphicolous larvae belonging to 5 genera, 6 species and 9 morpho-species of Melolonthidae. Species accumulation curves were obtained and the abundance per site, month and capture method was estimated. Comparing the beetle 
communities of tropical deciduous forest (BTC) and crop lands (ZA), 34 and 36 species and 603 and 417 individuals were found in each site respectively. Shannon's diversity index was 2.79 for ZA and 2.67 for BTC. Likewise, 82 larvae belonging to four genera were found in BTC, and 30 larvae of four genera in ZA. We select adults of 24 indicator species in BTC and 23 species in ZA, all from the subfamilies Dynastinae, Rutelinae and Scarabaeinae. The abundance of indicator species was 313 and 245 and the diversity index was 2.24 and 2.43 in ZA and BTC respectively. We conclude that the species composition of both communities is similar (49\%), and the effect of farming near the forest is negligible on the scarab beetle community.

Key words: Melolonthidae, Scarabaeidae, Trogidae, adults, larvae, Ecology, tropical deciduous forest.

\section{INTRODUCCIÓN}

La gran cantidad de relaciones biológicas que tienen las especies de Lamellicornia o Scarabaeoidea, su abundancia, diversidad, amplia distribución y buen nivel de conocimiento taxonómico, justifican plenamente las propuestas para emplearlos como indicadores ecológicos y biogeográficos durante estudios básicos o aplicados para evaluar el estado de equilibrio y conservación de los ecosistemas donde son abundantes, o para determinar el valor de importancia de una región como refugio faunístico, parque natural o reserva de la biosfera (Morón \& Aragón 2003). En el estado de Puebla, se han realizado trabajos faunísticos en el bosque de encinos de la Sierra del Tentzo (Morón et al. 2000) y en una zona agrícola de la región de Atlixco (Aragón et al. 2001), así como en los bosques húmedos de Cuetzalan en la Sierra Norte de Puebla (Carrillo-Ruiz \& Morón 2003). Sin embargo la fauna de Scarabaeoidea del bosque tropical caducifolio en el sur del estado es poco conocida, como ocurre en otras zonas del país, ya que sólo se han publicado resultados sobre algunos bosques tropicales caducifolios de Jalisco, Nayarit, Morelos, Quintana Roo y Yucatán (Morón et al. 1988; 1998, Morón 1990; Deloya et al. 1993; Reyes-Novelo \& Morón 2005).

Debido a las características biológicas de cada una de las subfamilias de Melolonthidae representadas en el Neotrópico, y al nivel de conocimiento sobre la taxonomía y distribución geográfica y ecológica de estos subgrupos, Morón (1997) recomendó el empleo de especies de Dynastinae y Rutelinae como indicadores de la diversidad en ambientes neotropicales. Por su parte, Favila y Halffter (1997) propusieron el uso de especies de la subfamilia Scarabaeinae como un grupo indicador para el estudio de aspectos básicos de la biodiversidad en bosques tropicales, y para la evaluación y el monitoreo de los efectos de las actividades humanas en estos ecosistemas.

Para el presente estudio se propuso hacer una comparación entre las comunidades de coleópteros Scarabaeoidea establecidas en la zona agrícola y el bosque tropical caducifolio del municipio de Santo Domingo Huehuetlán, Puebla, a fin de identificar especies características de la zona y determinar cuales de ellas pueden considerarse como indicadores ecológicos de diversidad. 


\section{DESCRIPCIÓN DEL ÁREA DE ESTUDIO}

El municipio de Santo Domingo Huehuetlán se encuentra ubicado al sur del Estado de Puebla (Fig. 1) entre los 18 $41^{\prime} 06^{\prime \prime}$ y $18^{\circ} 53^{\prime} 12^{\prime \prime}$ de latitud norte y entre los 98 $03^{\prime} 18^{\prime \prime}$ y $98^{\circ} 15^{\prime} 06^{\prime}$ " de longitud oeste, y tiene una superficie de $264 \mathrm{~km}^{2}$. Los límites del municipio son: al norte con el municipio de Puebla, al este con el municipio de Teopantlán, al sur con el municipio de Huatlatlauca y al oeste con los municipios de La Magdalena Tlatlauquitepec, San Juan Atzompa y Tzicatlacoyan. El municipio cuenta con 6,444 habitantes. Se tiene acceso a esta región a través de la carretera que va de San José El Aguacate a Santo Domingo Huehuetlán (Huehuetlán El Grande), que es la cabecera municipal. La topografía de la región es compleja, y la altitud de la zona de estudio varía entre los 1,300 y los 1,400 msnm.

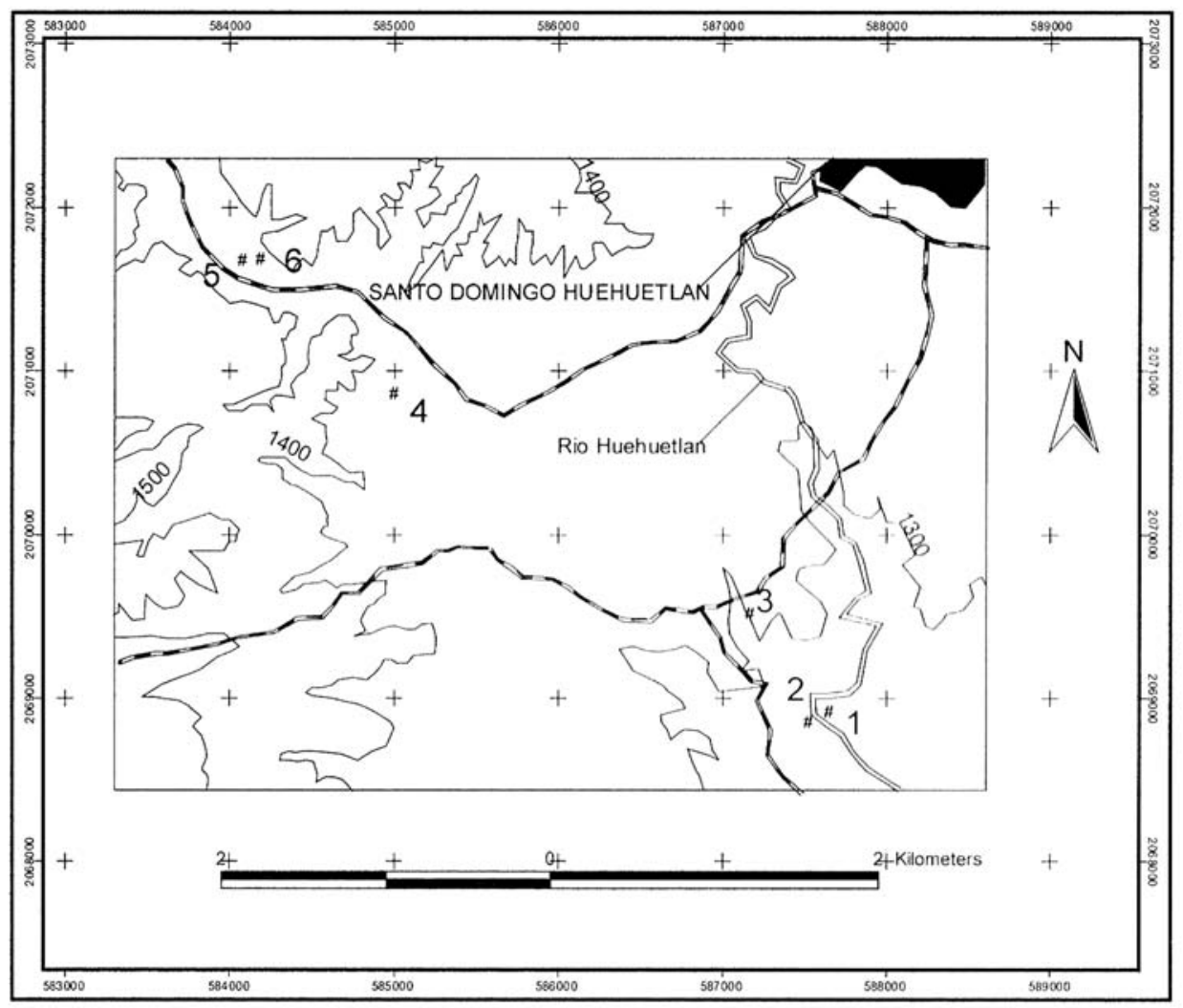

Figura 1. Ubicación de los sitios de trabajo al sur de municipio de Santo Domingo Huehuetlán, Pue. ( $1=$ TLM y CPT en ZA; $2=$ NTP en ZA; $3=$ NTP en ZA; 4= NTP en BTC; 5= TLM y NTP en BTC; $6=\mathrm{CPT}$ en BTC). 
Las unidades de suelo en la zona agrícola (ZA) corresponden a cambisol cálcico y castañozem háplico; y en el bosque tropical caducifolio (BTC) se presentan regosol cálcico, litosol y feozem háplico, y fase física lítica (capa rocosa a menos de $50 \mathrm{~cm}$ de profundidad). La geología en ZA corresponde a conglomerado (rocas sedimentarias) del cuaternario, y en BTC corresponde a esquisto (roca metamorfica) del paleozoico. El clima en la zona de estudio pertenece al grupo de los semicálidos (temperatura media anual entre $18^{\circ}$ y $22^{\circ} \mathrm{C}$; temperatura del mes más frío mayor de $18^{\circ} \mathrm{C}$ ) subhúmedos con lluvias en verano (precipitación del mes más seco menor de $60 \mathrm{~mm}$ ), subtipo de menor humedad dentro de los semicálidos subhúmedos (porcentaje de precipitación invernal menor de 5), es decir, $A(C) \mathrm{w}_{\mathrm{o}}(\mathrm{w})$. La vegetación y el uso del suelo en el área de estudio corresponde a bosque tropical caducifolio con vegetación secundaria y zona agrícola de temporal y de riego eventual. Se cultiva cacahuate, maíz, frijol, cebolla, "pipicha" (Porophyllum tagetoides D.C. Asteraceae), "pápalo quelite" (Porophyllum ruderale [Jacq.] Cass.), jitomate, tomate, calabacita, ejote, sandía, pepino y caña de azúcar; la ganadería que se practica sólo es de traspatio (INEGI 1994, 2000).

\section{MATERIAL Y MÉTODOS}

Se realizaron muestreos sistemáticos mensuales de adultos y larvas, diurnos y nocturnos entre octubre de 2005 y septiembre de 2006, en tres sitios seleccionados dentro del bosque tropical caducifolio (BTC) y tres sitios de la zona agrícola (ZA). En el Cuadro 1 se detalla la ubicación geográfica de los sitios de estudio y la posición de las trampas en estos sitios, de acuerdo con los registros de un geoposicionador satelital.

Para las colectas nocturnas de adultos se colocó una trampa con luz mercurial (TLM) tipo pantalla (Morón \& Terrón 1988) de 120 watts alimentada con un generador de electricidad portátil operada de las 20:00 a las 22:00 hrs. Los ejemplares capturados fueron sacrificados en frascos con vapores de acetato de etilo. Se separaron las muestras en períodos de media hora en frascos con alcohol etílico al $70 \%$. Además se instalaron en el suelo dos necrotrampas permanentes NTP-80 (Morón \& Terrón 1984) en cada sitio, separadas 1000 m entre sí, las cuales se revisaron durante el día cada quince días. Se colocó una coprotrampa (CPT) en cada sitio, la cual se activaba un día con $100 \mathrm{~g}$ de heces humanas frescas y se recogía en $24 \mathrm{hrs}$, los ejemplares se colocaron en frascos con alcohol al 70\%. En forma paralela se realizaron colectas directas dentro y bajo troncos derribados, bajo excremento de ganado, en el suelo, sobre follaje y vegetación y bajo hojarasca acumulada. Los ejemplares adultos se prepararon y montaron por los métodos convencionales.

Colecta de larvas. Para determinar en forma preliminar la abundancia, distribución vertical y los estados de desarrollo de los coleópteros escarabeoideos en el suelo, se obtuvieron muestras una vez al mes entre octubre de 2005 y septiembre 
de 2006. En el BTC se cavaron cinco pozos de 30x30x30 cm en disposición cuadrangular con uno al centro, separados $2 \mathrm{~m}$ entre sí. En el caso de ZA se tomaron 10 muestras de suelo de 50x50x50 cm en hileras de cuatro, separadas 20 m entre sí y a 10 metros de la orilla de la parcela agrícola (milpa). Cada mes se recorrieron estas hileras un metro de distancia para no coincidir con ocasiones anteriores. Todos los ejemplares inmaduros se fijaron en líquido de Pampel (Morón \& Terrón 1988).

Los adultos se determinaron con ayuda de las claves de Deloya et al. (1995), Morón et al. (1998, 2000) y Pérez-García (1999), y las larvas se identificaron con las claves propuestas por Morón (1983) y Morón \& Deloya (1991). Se siguió un sistema de clasificación supragenérica modificado a partir de Endrödi (1966), Morón et al. (1997) y Morón (2003). Los ejemplares se depositaron en las colecciones del Instituto de Ecología, A.C. (IEXA), del Departamento de Agroecología y Ambiente del ICUAP (DAGAM), y de la Escuela de Biología de la BUAP (EBBUAP).

Cuadro 1. Ubicación geográfica (en UTM y msnm) de los sitios de estudio y de los diferentes tipos de trampas en los mismos. BTC: bosque tropical caducifolio; ZA: Zona agrícola.

\begin{tabular}{lcccc}
\hline Atrayentes & Sitios & \multicolumn{3}{c}{ Coordenadas de los sitios de estudio } \\
& & & & \\
& & & & \\
& & & & \\
Trampa de luz (TLM) & BTC 1 & 14584083 & 2071676 & 1368 \\
& ZA & 14587661 & 2068911 & 1297 \\
Necrotrampas (NTP) & ZA 1 & 14587535 & 2068852 & 1294 \\
& ZA 2 & 14587179 & 2069518 & 1307 \\
& BTC 1 & 14585010 & 2070858 & 1364 \\
& BTC2 & 14584195 & 2071687 & 1382 \\
Coprotrampas (CPT) & ZA & 14587661 & 2068911 & 1297 \\
& BTC & 14584083 & 2071676 & 1368 \\
\hline
\end{tabular}

Se hizo una síntesis de los datos de captura, contabilizando los individuos y las especies por mes, por sitio y por tipo de muestreo. Se aplicaron pruebas de hipótesis ("chi cuadrada" y "t de student") para detectar diferencias en las abundancias totales y en el número de especies (riqueza) por sitio y por método de muestreo. Se estimaron la diversidad alfa, beta y gama, así como la dominancia y la complementaridad. En el caso de la diversidad alfa, para establecer cual es la relación entre el número de especies nuevas acumuladas y el esfuerzo de captura, se aplicó el Modelo de Dependencia Lineal (Soberón \& Llorente 1993). Para entender la relación entre abundancia y riqueza, se calculó el Índice y la equitabilidad de Shannon-Wiener (Magurran 1988). Una prueba de " $t$ " permitió comparar la diversidad de ambos sitios (Magurran 1988). Para estimar la dominancia, se calculó el 
Índice de Simpson (Moreno 2001). En el caso de la diversidad beta se empleó el Coeficiente de Similitud de Jaccard y la complementaridad, mientras que la diversidad gamma se obtuvo desarrollando el cálculo basado en el índice de Shannon (Moreno 2001).

Indicadores de biodiversidad. A partir de la lista de especies en cada localidad, se seleccionaron como especies indicadoras de biodiversidad aquellas pertenecientes a las subfamilias Dynastinae y Rutelinae de acuerdo con Morón (1997), y aquellas pertenecientes la subfamilia Scarabaeinae según Favila \& Halffter (1997).

\section{RESULTADOS}

\section{Especies de Scarabaeoidea presentes en Santo Domingo Huehuetlán, Puebla}

Se colectaron 1,020 ejemplares de adultos representantes de 52 especies incluidas en 29 géneros de tres familias de Scarabaeoidea, y 113 larvas pertenecientes a cinco géneros, seis especies y nueve morfoespecies de Melolonthidae. Phyllophaga fulviventris, P.etabatesiana, Polyphylla petiti, Cyclocephala ovulum, Copris lecontei y Pseudocanthon perplexus se registran por primera vez en el estado de Puebla.

\section{Melolonthidae, Melolonthinae (Cuadro 2)}

Diplotaxis Kirby, 1837. Se obtuvieron adultos de tres especies: D. angularis: 31 machos y 73 hembras en BTC y 9 machos y 11 hembras en ZA; todos atraídos por la TLM entre 20:00-22:30 hrs, excepto dos hembras en NTP durante abril y mayo. D. marginicollis: un macho y cinco hembras en BTC y un macho y dos hembras en ZA, todos en TLM entre 20:00-22:00 hrs. D. mediafusca: un macho en ZA en TLM entre 21:30-22:00 hrs. Estados inmaduros (Cuadro 6). Diplotaxis sp. 1: 27 larvas de segundo estadio (L2) en BTC y una en ZA; 2 larvas de tercer estadio (L3) en BTC y seis en ZA. Diplotaxis sp. 2: una L2 y una L3 en BTC.

Phyllophaga Harris, 1827. Se capturaron adultos de ocho especies (Cuadro 2): $P$. brevidens: una hembra en BTC, 27 machos y 28 hembras en ZA; todos atraídos por TLM entre 20:00-20:30 hrs, excepto una hembra en NTP y otra sobre carrizos durante junio, y dos machos sobre el suelo en mayo. P. fulviventris: un macho en ZA atraído por TLM entre 21:00-21:30 hrs. P. ilhuicaminai: 23 hembras en BTC, 40 machos y 49 hembras en ZA; todos atraídos por TLM entre 20:00-21:30 hrs, excepto dos hembras y tres machos sobre carrizos durante mayo. $P$. ravida: cuatro hembras en BTC, seis hembras en ZA; siete de ellas atraídas por TLM entre 20:00-21:30 hrs, dos en NTP y otra sobre el suelo en septiembre. P. setifera: 50 hembras y 19 machos en ZA atraidos por TLM entre 20:30-21:30 hrs, excepto tres hembras capturadas en el suelo durante mayo. P. (Listrochelus) sp.: tres hembras y dos machos en BTC, dos hembras y un macho en ZA, atraídos por TLM entre 20:00-21:30 hrs. P. (Chlaenobia) etabatesiana: un macho en BTC atraído por TLM entre 20:30-21:00 hrs. P. (Phyllophaga) sp. tres hembras en ZA atraídas por TLM entre 20:30-22:00 hrs. Estados inmaduros (Cuadro 6). P. ilhuicaminai: cuatro larvas de tercer estadio (L3) 
en BTC; cuatro larvas de segundo estadio (L2) y tres L3 en ZA. P. etabatesiana: cuatro larvas de primer estadio (L1), 12 L2 y seis L3 en BTC. P. setifera: una L3 en BTC. P. ravida: una L2 y 11 L3 en ZA. Phyllophaga sp.: una L1 en BTC.

Polyphylla Harris, 1841. Solo se obtuvo un macho de P. petiti en ZA durante diciembre, atraído por TLM entre 21:00-21:30 hrs.

\section{Melolonthidae, Rutelinae (Cuadro 2)}

Paranomala Casey, 1915. Se colectaron representantes de cuatro especies: P. chevrolati: una hembra en BTC y tres parejas en ZA, atraídos por TLM entre 20:30-21:30 hrs. $P$. cincta: tres hembras en BTC y 10 hembras y 8 machos en ZA, todos atraídos por TLM entre 20:00-22:00 hrs. P. flavilla: dos hembras en ZA en TLM 20:30-22:00 hrs. P. villosela: una pareja en ZA sobre el suelo en octubre. Estados inmaduros (Cuadro 6). Paranomala sp.1: dos L3 en BTC. Paranomala sp. 2: seis L2 en BTC. Paranomala sp. 3: una L3 en BTC. Paranomala sp. 4: una L3 en BTC. Paranomala sp. 5: una L2 y tres L3 en ZA.

Pelidnota Mac Leay, 1819. Se obtuvieron siete machos y nueve hembras de $P$. virescens en ZA atraídos por la TLM entre 20:30-22:30 hrs.

\section{Melolonthidae, Dynastinae (Cuadro 2).}

Cyclocephala Latreille, 1829. Se colectaron representantes de tres especies: $C$. lunulata: cuatro hembras y dos machos en BTC y 50 hembras y 43 machos en ZA, la mayoría atraídos por TLM entre 20:30-22:00 hrs, excepto un macho en NTP durante febrero y otro macho en CPT durante noviembre. C. melanocephala: dos hembras en ZA atraídas por TLM entre 20:00- 21:30 hrs. C. ovulum: una hembra en ZA atraída a TLM entre 20:00-20:30 hrs. Estados inmaduros (Cuadro 6). Cyclocephala lunulata: una L3 en ZA.

Dynastes Kirby, 1825. Se obtuvieron dos hembras de D. hyllus en BTC atraídas por TLM entre 21:00-22:30 hrs.

Strategus Hope, 1837. Fue posible capturar cinco hembras de $S$. aloeus en ZA y otra hembra en BTC, atraídas por TLM entre 20:30-22:00 hrs.

Xyloryctes Hope, 1837. Solo se colectaron dos hembras de X. telephus en BTC. Una en TLM entre 21:00-21:30 hrs y otra en NTP.

Ligyrus Burmeister, 1847. Tres hembras de L. sallei en BTC y 18 hembras en ZA, una sobre el suelo, dos en NTP y las restantes en TLM entre 20:00-22:00 hrs.

Phileurus Latreille, 1807. Se obtuvieron dos hembras de P. valgus en ZA atraídas por TLM entre 20:30-21:00 hrs.

Melolonthidae, Cetoniinae (Cuadro 2)

Euphoria Burmeister, 1842. Se colectaron 12 machos y 13 hembras de E. basalis en ZA dentro de flores de Cucurbita sp. (Cucurbitacea). durante octubre. Estados inmaduros (Cuadro 6). Euphoria basalis: tres L2 en BTC. 
Yanez-Gómez \& Morón: Fauna de Scarabaeoidea de Huehuetlán, Puebla

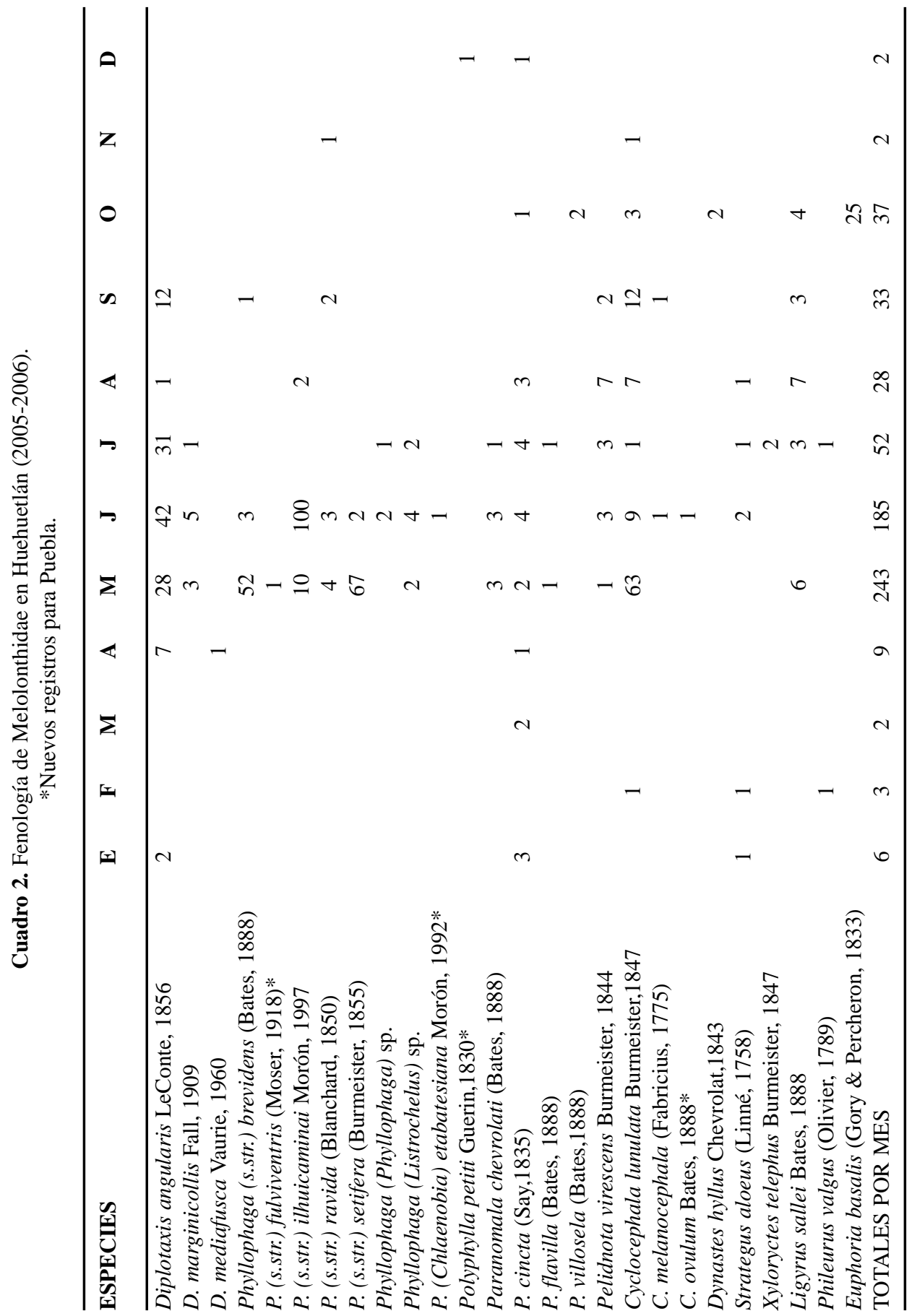




\section{Scarabaeidae (sensu stricto), Scarabaeinae (Cuadro 3)}

Ateuchus Weber, 1801. Se obtuvieron dos parejas de A. rodriguezi en BTC, tres de ellos en CPT y otro en el suelo; mientras que en ZA se encontraron dos machos y cuatro hembras, dos de ellos en CPT y cuatro en TLM entre 21:30- 22:30 hrs.

Canthidium Erichson, 1847. En BTC se colectaron seis hembras y un macho de C. laetum, cuatro sobre suelo, una en NTP y una en TLM entre 20:00-20:30 hrs, y uno en CPT; en ZA se encontraron dos hembras en CPT.

Copris Müller, 1764. Se encontraron representantes de tres especies: $C$. halffteri: cinco hembras y un macho en BTC, tres de éstos en TLM entre 21:00-22:00 hrs, una en excremento caprino, una en excremento vacuno y una en CPT. C. lecontei: ocho hembras, cuatro en NTP de ZA y cuatro en BTC, dos de ellas en TLM entre 21:3022:00 hrs, una en NTP y otra en excremento de vacuno. C. rebouchei: un macho en TLM de BTC entre 20:30-21:00 hrs, y tres en excrementos de vacuno, caprino y equino en ZA.

Dichotomius Hope, 1838. Se capturaron representantes de dos especies: D. amplicollis: cuatro machos y ocho hembras en BTC, nueve en CPT, dos en NTP, una en TLM entre 20:00-20:30 hrs y en ZA sólo se colectó un macho en TLM entre 22:00-22:30 hrs. D. colonicus: dos hembras en TLM de ZA y una pareja en TLM de BTC entre 21:30-22:00 hrs.

Euoniticellus Janssens, 1953. Se obtuvieron seis hembras y 11 machos de E. intermedius en BTC, nueve en excremento vacuno, dos en CPT, seis en TLM entre 21:00-21:30 hrs.

Digitonthophagus Balthasar, 1959. En BTC se colectaron 16 hembras y siete machos de D. gazella, ocho en excremento vacuno, una en CPT y 14 en TLM entre 20:30-22:30 hrs y tres hembras en ZA atraídas por TLM entre 20:00-22.00 hrs.

Onthophagus Latreille, 1802. Fue posible reunir representantes de cinco especies: O. hoepfneri: un macho en excremento vacuno en BTC. O. lecontei: dos hembras y cinco machos en BTC, tres en excremento vacuno, dos en CPT y dos en NTP. O. rostratus: un macho en CPT de BTC y una hembra en NTP de ZA. $O$. rufescens: cuatro hembras y tres machos en CPT de BTC. O. mexicanus: seis machos y 12 hembras en CPT de BTC.

Coprophanaeus Olsoufieff, 1924. Se registraron 13 hembras y 22 machos de $C$. pluto en BTC en NTP excepto una en TLM entre 21:00-21:30 hrs, mientras que en ZA se capturaron 37 hembras y 39 machos, todos en NTP excepto dos en TLM entre 20:30-21:30 hrs.

Phanaeus MacLeay, 1819. En BTC se obtuvieron cuatro hembras y tres machos de $P$. daphnis, dos en excremento vacuno, tres en excremento caprino, uno en CPT y otro sobre el suelo.

Canthon Hoffmannsegg, 1817. Se obtuvieron representantes de dos especies: $C$. corporaali: en BTC se colectaron 28 hembras y 24 machos, 40 en CPT y 12 en NTP, 
mientras que en ZA se registraron 10 hembras y cinco machos, una sobre suelo, cinco en CPT y nueve en NTP. C. indigaceus chevrolati: en BTC se capturaron 114 hembras y 27 machos, 90 directamente sobre el suelo en milpa abonada con excremento caprino, 41 colectadas sobre boñiga de ganado vacuno, dos en CPT y ocho en NTP; mientras que en ZA se obtuvieron seis hembras en NTP.

Pseudocanthon Bates, 1887. Con CPT se colectaron seis hembras y 10 machos de P. perplexus en ZA.

Deltochilum Eschscholtz, 1822. En BTC se registraron 42 hembras y 12 machos de D. gibbosum sublaeve, tres en CPT y 51 en NTP, mientras que en las NTP situadas en ZA se capturaron 15 hembras.

\section{Scarabaeidae, Aphodiinae (Cuadro 3)}

Labarrus Mulsant \& Rey, 1870. En BTC se encontraron 15 ejemplares de $L$. pseudolividus, la mayoría en TLM entre 20:30-21:00 hrs y uno sobre el suelo en enero, mientras que en ZA se capturaron cuatro individuos en TLM durante el mismo horario. Es una especie identificada durante muchos años como Aphodius (Labarrus) lividus (Olivier, 1789), o Labarrus lividus, nombre que en realidad corresponde a un taxón introducido de Europa en algunas regiones de América.

Platytomus Mulsant, 1842. En BTC se colectaron 90 ejemplares de Platytomus micros atraídos por la TLM entre 20:30-21:00 hrs, mientras que en ZA se obtuvieron 220 ejemplares en TLM durante el mismo horario. Cabe mencionar que tanto esta especie como L. pseudolividus acudieron en grandes cantidades a la TLM pero solo se recolectaron muestras representativas.

\section{Ochodaeidae, Ochodaeinae (Cuadro 4).}

Ochodaeus Dejean, 1821. Solo se encontró un ejemplar de O. howdeni atraído a TLM en ZA entre 20:00- 20:30 hrs.

\section{Hybosoridae, Hybosorinae (Cuadro 3)}

Hybosorus MacLeay, 1819. En BTC se capturaron dos machos y una hembra de $H$. illigeri en TLM entre 20:00-21:30 hrs, mientras que en ZA se obtuvieron dos hembras y un macho en TLM entre 21:30- 22:30 hrs.

\section{Trogidae (Cuadro 3)}

Omorgus Erichson, 1847. Se registraron dos especies: O. suberosus: 11 hembras en BTC, cinco en NTP y seis en TLM entre 20:00-22:00 hrs, mientras que en ZA cuatro hembras y seis machos, cinco en NTP y cinco en TLM. O. tesselatus: en ZA seis hembras y dos machos, cinco en NTP y tres en TLM entre 20:00-21:00 hrs. 
Acta Zoológica Mexicana (n.s.) 26(1) (2010)

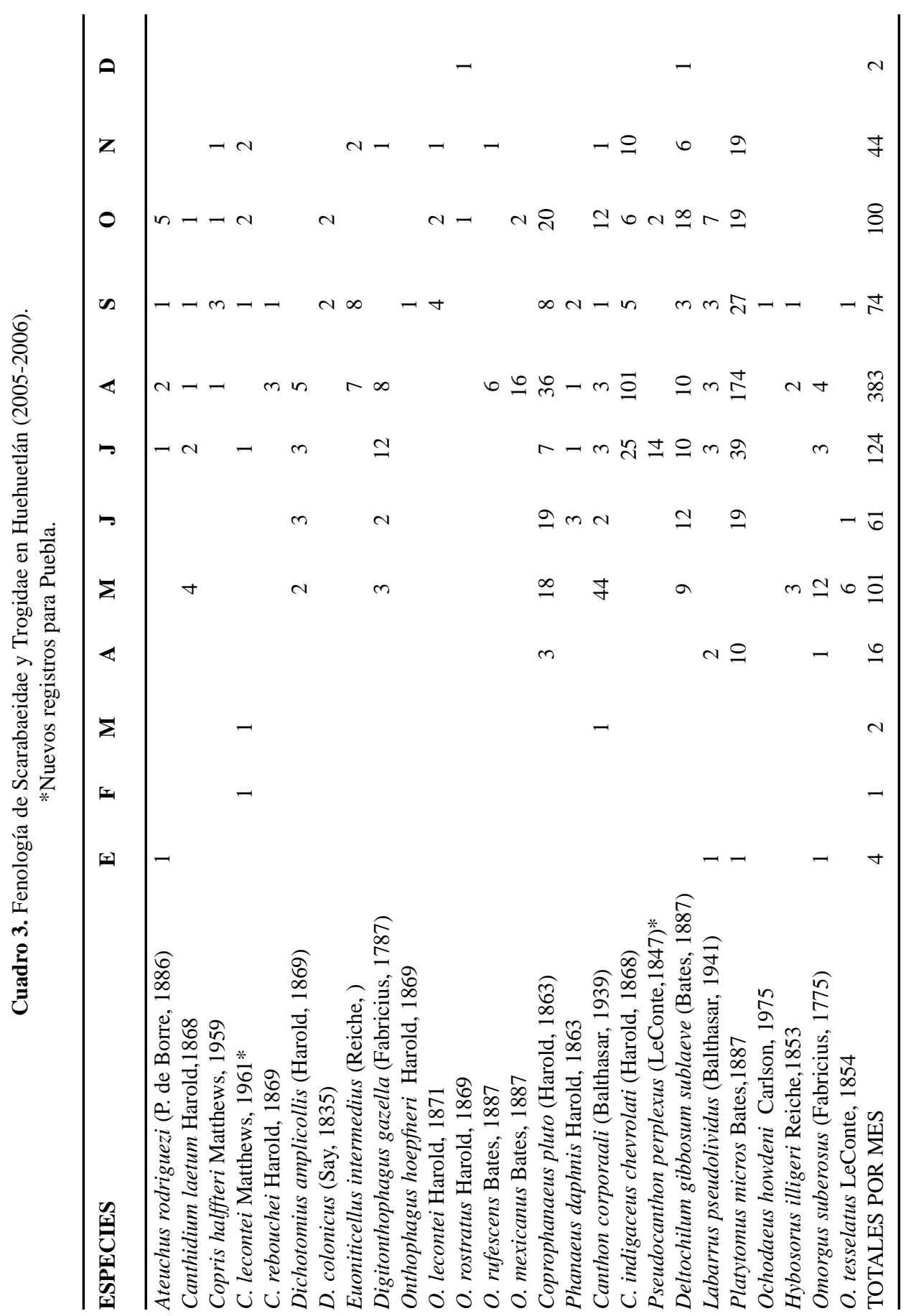




\section{Composición de especies}

Las especies obtenidas por colecta directa no se incluyeron en los análisis comparativos: en ZA Anomala villosela, Euphoria basalis y Labarrus pseudolividus; en BTC Onthophagus hoepfneri, Labarrus pseudolividus y Platytomus micros. Aunque L. pseudolividus y P. micros fueron capturadas en trampa de luz, se incluyen en colecta directa ya que su captura no fue sistemática, ni el esfuerzo de captura fue el mismo en los dos sitios; por su pequeño tamaño y gran abundancia sólo se recolectó una muestra de todos los individuos disponibles en las trampas.

De las otras 47 especies encontradas en los dos sitios, 23 son compartidas (48.93\%), 13 son exclusivas de ZA (27.6\%) y 11 son exclusivas de BTC (23.4\%). El total de especies podría aumentar cuando menos a 69 taxones con base en las colecciones DAGAM, EBBUAP e IEXA que mantienen muestras de otras recolectas parciales de Scarabaeoidea en el municipio de Huehuetlán. En cuanto a larvas, el esfuerzo de captura no fue el mismo entre los dos ambientes, por lo cuál no se realizaron comparaciones directas de riqueza de larvas entre los dos sitios. Solo se refiere la presencia de larvas de cuatro géneros y 12 morfoespecies en BTC, y de larvas de cuatro géneros y seis morfoespecies en ZA.

\section{Curva de acumulación de especies}

En primer lugar se estimó el esfuerzo de captura total (en horas de muestreo) para los dos sitios de estudio (excluyendo colecta directa); asimismo, dividiendo estos valores entre dos obtenemos el esfuerzo de captura por sitio (Cuadro 4). Cabe mencionar que para obtener la curva acumulativa de especies se empleó el Modelo de Dependencia Lineal, ya que según Soberón \& Llorente (1993), es un modelo adecuado cuando se muestrean taxones bien conocidos en áreas pequeñas con pocas especies raras. Los parámetros del Modelo de Dependencia Lineal son la correlación (0.9992 para ZA y 0.9977 para BTC) y la asíntota (38.24 para ZA y 35.33 para BTC) que representa el número máximo de especies esperado. La eficiencia del muestreo con base en el modelo empleado, fue de $94.14 \%$ para ZA y $96.23 \%$ para BTC, y el número de especies faltantes fue de dos en ZA y una en BTC.

\section{Riqueza}

En ZA hay más especies en mayo (21) y junio (22) y menos especies en enero (1) y febrero (1); en BTC hay más especies en julio (12) agosto (12) y septiembre (16) y menos en febrero (0) y marzo (0). Se observa un mayor número de especies en ZA (36) que en BTC (34). Para confirmar esta diferencia se aplicó una prueba de hipótesis (Chi cuadrada), en la que se obtuvo una $\mathrm{p}=0.8110$, por lo que se concluye que los valores de riqueza no difieren significativamente. 


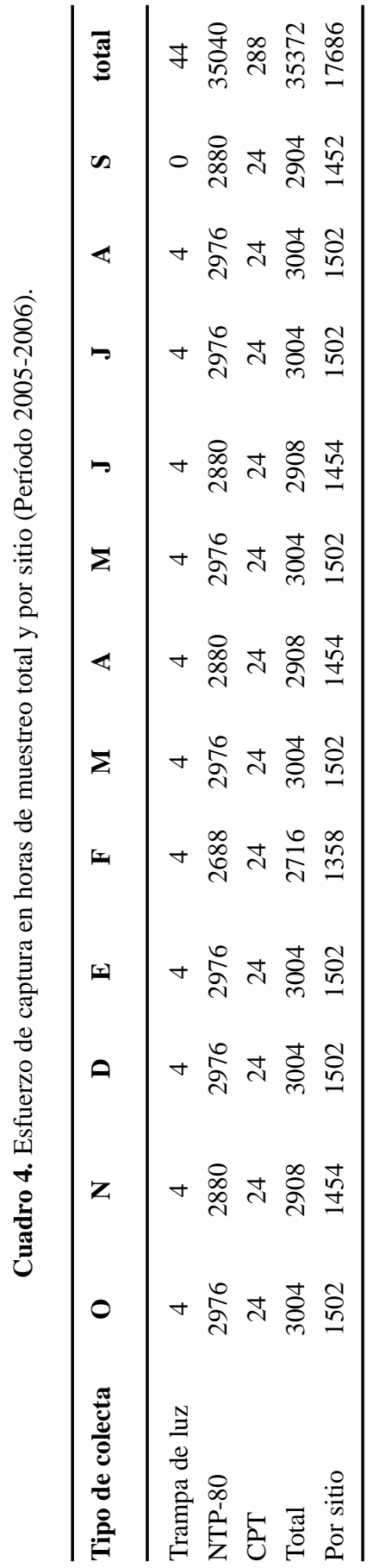

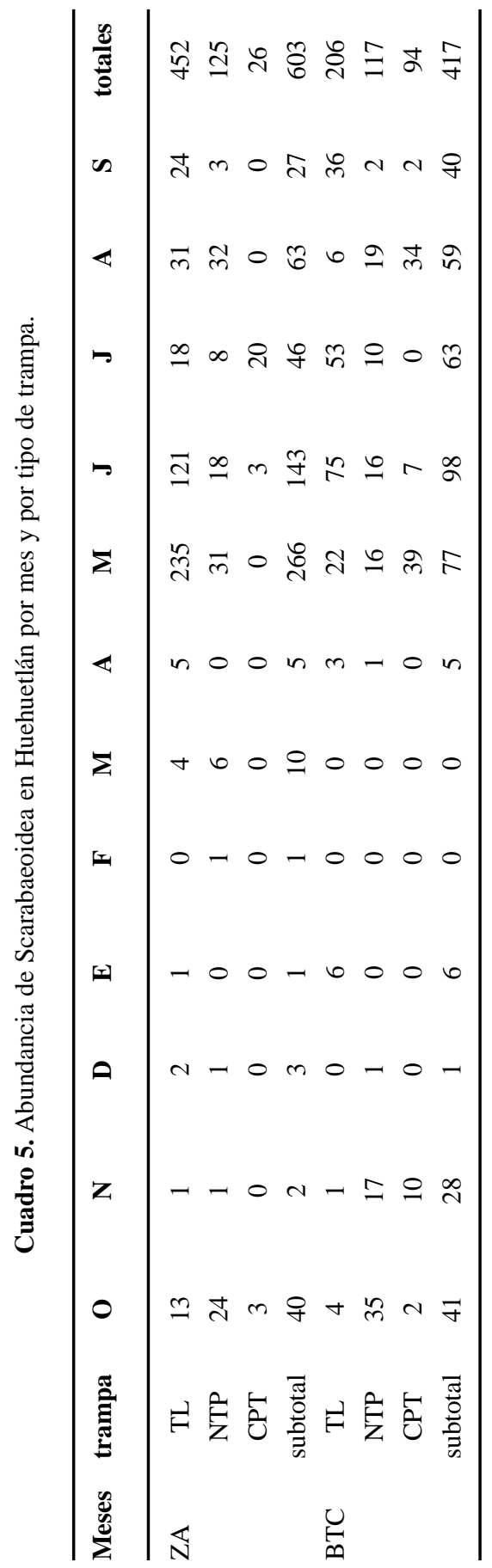




\section{Abundancia}

Adultos. En el Cuadro 5 se desglosa la abundancia por sitio, por mes y por método de colecta $(\mathrm{TLM}=$ trampa de luz, $\mathrm{NTP}=$ necrotrampa, $\mathrm{CPT}=$ coprotrampa $) . \mathrm{Se}$ aprecia una mayor abundancia de ejemplares capturados en TL, seguida de NTP y CPT, resultando en una abundancia total mayor en ZA que en BTC. En el caso de las CPT se capturaron más individuos en BTC que en ZA. Es mayor la abundancia total en ZA (603) que en BTC (417), para lo cual se aplicó una prueba de Hipótesis (Chi cuadrada) para probar la hipótesis nula (Ho) entre los valores de abundancia total en ambos sitios de trabajo, y se obtuvo un resultado de $\mathrm{p}=5.7495 \mathrm{E}-09$, por tanto existen diferencias significativas entre ambas zonas.

Se observó que las especies de Melolonthidae fueron mas abundantes en ZA $(71.8 \%)$ que en BTC (41.2\%), mientras que la mayor abundancia de Scarabaeidae se registró en BTC (56.1\%) y los Trogidae fueron un poco mas abundantes en ZA (3\%). En concordancia con los resultados anteriores, a nivel de subfamilia se aprecia mayor abundancia de Melolonthinae en ZA (44.4\%) y Scarabaeinae en BTC (55.4\%). En BTC hay más especies raras, por lo que es mayor la dominancia de una sola especie (Diplotaxis angularis $25.18 \%$, seguida de Canthon corporaali $13.43 \%$ y Deltochilum gibbosum $12.95 \%$ ). En ZA no hay tal patrón, ya que por lo menos cinco especies son abundantes (Cyclocephala lunulata 14.90\%, Phyllophaga ilhuicaminai $13.91 \%$, Coprophanaeus pluto $13.08 \%$, P. setifera $11.09 \%$ y P. brevidens $9.44 \%$ ).

Larvas. En los dos sitios de estudio se encontraron patrones semejantes (Cuadro 6). Las larvas más abundantes en BTC representan al género Phyllophaga, seguidas de Diplotaxis, Paranomala y Euphoria. En ZA las larvas más abundantes corresponden a Phyllophaga, seguidas de Paranomala, Diplotaxis y Ligyrus. En cuanto a la abundancia de larvas por mes se observó una baja abundancia en primavera, seguida de una marcada escasez a principios del verano y un notable aumento en septiembre. En otoño disminuye paulatinamente la abundancia hasta llegar a cero en invierno.

\section{Diversidad y Dominancia para adultos de Scarabaeoidea en ambos sitios de estudio}

Diversidad alfa. El Índice de Diversidad de Shannon Wiener para ZA es de 2.79 y para BTC es de 2.67; por medio de una prueba de hipótesis ( $t$ de Student) se aprecia que no hay diferencia entre estos valores para ambos sitios ( $p>0.05)$. Por otro lado, dado que este índice puede tomar valores entre cero y el logaritmo de $\mathrm{S}$ (3.58 en ZA y 3.52 en BTC), se pueden considerar como valores relativamente altos de diversidad alfa en general en cada sitio. El Índice de Dominancia de Simpson, que se puede interpretar como el inverso de la equitabilidad, es mayor en BTC (0.11) que en ZA (0.09). La Equitabilidad de Shannon- Wiener fue de 0.7810 en ZA y de 0.7572 en BTC. 
Cuadro 6. Fenología y abundancia de larvas de Melolonthidae en Huehuetlán (2005-2006)

\begin{tabular}{|c|c|c|c|c|c|c|c|c|c|c|c|c|}
\hline Taxones & $\mathbf{M}$ & $\mathbf{A}$ & $\mathbf{M}$ & $\mathbf{J}$ & $\mathbf{J}$ & $\mathbf{A}$ & $\mathbf{S}$ & $\mathbf{O}$ & $\mathbf{N}$ & D & $\mathbf{Z A}$ & BTC \\
\hline Diplotaxis sp. 1 & & & & & & & 24 & 11 & & 1 & 7 & 29 \\
\hline Diplotaxis sp. 2 & & & 2 & & & & & & & & & 2 \\
\hline Phyllophaga ravida & & & 2 & & 2 & & 4 & 1 & 1 & 2 & 12 & \\
\hline P. ilhuicaminai & & & & & & & 8 & 3 & & & 7 & 4 \\
\hline P. etabatesiana & 3 & 2 & 4 & & & & 8 & 4 & 1 & & & 22 \\
\hline P. setifera & 1 & & & & & & & & & & & 1 \\
\hline Phyllophaga sp. & & & & & & & 1 & & & & & 1 \\
\hline Melolonthinae sp. & & 1 & & & & & 7 & 2 & & & 3 & 8 \\
\hline Paranomala sp. 1 & & & & & & & 2 & & & & & 2 \\
\hline Paranomala sp. 2 & & & & & & & 5 & 1 & & & & 6 \\
\hline Paranomala sp. 3 & & & & & & & & 1 & & & & 1 \\
\hline Paranomala sp. 4 & & 1 & & & & & & & & & & 1 \\
\hline Paranomala sp. 5 & & 2 & 1 & & & & 1 & & & & 4 & \\
\hline Cyclocephala lunulata & & & & & & & & & & 1 & 1 & \\
\hline Euphoria basalis & & & & & & & 3 & & & & & 3 \\
\hline
\end{tabular}

Diversidad Beta. Dado que el Coeficiente de similitud de Jaccard es igual a 0.49, puede decirse que las comunidades son semejantes en un $49 \%$ (o diferentes en un $51 \%$, según el valor de Complementariedad).

Diversidad Gamma. Con base en el Índice de Shannon, la diversidad gamma fue de 3.044; se estimó la aportación de la diversidad alfa promedio $(2.73=89.68 \%)$ y la diversidad beta $(0.314=10.32 \%)$ a la diversidad gamma.

Cuadro 7. Abundancia y riqueza de especies indicadoras en BTC y ZA

\begin{tabular}{lcccccc}
\hline Ambiente & Atributo & Abundancia & Abun.Rel.(\%) & Dynastinae & Rutelinae & Scarabaeinae \\
& & & & & & \\
\hline BTC & abundancia & 245 & 100.0 & 11 & 6 & 228 \\
& riqueza & 23 & & 3 & 5 & 15 \\
\multirow{2}{*}{ ZA } & abundancia & 313 & 100.0 & 123 & 42 & 148 \\
& riqueza & 21 & & 6 & 4 & 11 \\
\hline
\end{tabular}

\section{Especies indicadoras}

En el Cuadro 7 se muestra la riqueza y la abundancia de especies indicadoras (subfamilias Dynastinae, Rutelinae y Scarabaeinae) en ambos sitios. En BTC las especies indicadoras dominantes son Canthon corporaali (22.8\%) y Deltochilum gibbosum (22\%), y en ZA son Cyclocephala lunulata (28.7\%) y Coprophanaeus 
pluto (25.2\%). Al emplear especies indicadoras se aprecia una relación inversamente proporcional entre diversidad (riqueza) y abundancia (dominancia). Sin embargo no hay diferencia significativa entre los valores de riqueza ni de diversidad (Cuadro 8).

Cuadro 8. Atributos de las comunidades de especies indicadoras en ZA y BTC

\begin{tabular}{lcccc}
\hline ATRIBUTOS & ZA & BTC & p & Estadígrafo \\
\hline Riqueza & 21 & 23 & 0.7630 & Chi Cuad. \\
Abundancia* & 313 & 245 & 0.0040 & Chi Cuad. \\
Indice Diversidad S.W. & 2.24 & 2.43 & $>0.05$ & T de Student \\
Equitabilidad & 0.7387 & 0.7756 & - & - \\
Indice Dominancia de Simpson & 0.1661 & 0.1310 & - & - \\
\hline
\end{tabular}

* significativo

Cuadro 9. Coeficientes de correlación (r) entre abundancia y riqueza de Scarabaeoidea y precipitación y temperatura en Huehuetlán. (Abundancia en número de individuos).

RELACION r r

Precipitación (mm) vs número de especies (S) en ZA

Precipitación (mm) vs Abundancia en ZA

0.38

Precipitación (mm) vs número de especies (S) en BTC

$0.82 *$

Precipitación vs Abundancia en BTC

Temperatura $\left({ }^{\circ} \mathrm{C}\right)$ vs número de especies $(\mathrm{S})$ en ZA

Temperatura $\left({ }^{\circ} \mathrm{C}\right)$ vs Abundancia en ZA

$0.68^{*}$

Temperatura $\left({ }^{\circ} \mathrm{C}\right)$ vs número de especies $(\mathrm{S})$ en BTC

0.18

Temperatura $\left({ }^{\circ} \mathrm{C}\right)$ vs Abundancia en BTC

$0.57 *$

*significativo

\section{Riqueza y Abundancia}

\section{DISCUSIÓN}

En una primera etapa, se analizan algunos atributos de las comunidades (riqueza y abundancia) empleando todas las especies capturadas con trampas, y en un segundo análisis sólo se emplean las especies indicadoras (en el sentido de Morón 1997 y Favila \& Halffter 1997). En ZA se encontraron 36 especies, y en BTC 34; del total de las 47 especies encontradas en ambos sitios se comparten 23. Esto indica que hay 
mucha semejanza en ambos lugares como lo indica el Índice de Jaccard (0.49, es decir, 49\%). Se buscó que los sitios tuvieran las mismas características geográficas en general (mismo clima y cercanía geográfica), pero diferente tipo de vegetación y uso del suelo (bosque tropical caducifolio y zona agrícola), para detectar un efecto antrópico y mantener las demás variables constantes. La similitud en la composición específica es un reflejo de la cercanía geográfica, pero las especies únicas de cada sitio (13 en ZA y 11 en BTC) son un reflejo del tipo de uso del suelo, lo que se aprecia mejor en los resultados con las especies indicadoras.

En términos de composición de especies, la localidad de muestreo presenta similitudes marcadas con las faunas de Chamela, Jalisco (Morón et al.1988) y Jojutla, Morelos (Deloya \& Morón 1994), en virtud de los parecidos en el clima y el tipo de vegetación. Las tres localidades presentan clima cálido subhúmedo con lluvia en verano (Aw) y vegetación de bosque tropical caducifolio. En cuanto a la composición de especies en estado larvario, se obtuvieron 15 especies en BTC y ocho en ZA. No se pueden hacer comparaciones directas de riqueza de larvas en ambos sitios dado que el esfuerzo de captura fue diferente, pero se puede comparar la similitud entre morfoespecies en trabajos posteriores.

Se llevaron a cabo 24 muestreos sistemáticos mensuales a lo largo de un año, lo que nos permitió estimar las curvas acumulativas de especies. En este sentido se aplicó el Modelo de Dependencia Lineal (Soberón \& Llorente 1993; Moreno 2001) y se obtuvo una eficiencia en el muestreo del $94.14 \%$ (en ZA) y $96.24 \%$ (en BTC), obteniendo 38 y 35 especies esperadas respectivamente. Este resultado es exclusivo para estos sitios con los métodos empleados en este trabajo. Se han observado porcentajes de eficiencia en muestreos de Scarabaeoidea con trampa de luz y necrotrampas del 72\% al 86\% (García de Jesús 2006) y del 78\% al 86\% (SalamancaCalixto 2006) en ambientes templados y semifríos; y con Scarabaeinae en trampas de caída, coprotrampas y necrotrampas, del $86 \%$ al 94\% (Favila 2005) en ambientes calido- húmedos. Cabe mencionar que se aplicaron modelos no paramétricos (Bootstrap, Jacknife en los primeros dos trabajos, y MMMean en el tercer trabajo) diferentes al empleado en este trabajo para ajustar las curvas de acumulación de especies.

La riqueza de especies a lo largo del año sigue un patrón estacional en la zona agrícola, el cual es muy semejante a los resultados de Morón (1994) para Scarabaeoidea de Hidalgo. Este patrón, en general, se puede atribuir a la mayor disponibilidad de recursos en verano como resultado de la productividad de los ambientes, por efecto de la precipitación y la temperatura (aunque no existe una correlación estrecha de la riqueza y la abundancia con la temperatura y la precipitación a lo largo del año; ver Cuadro 9). Las diferencias entre ZA y BTC (estacionalidad menos marcada en BTC) se debe a la presencia y actividad de más especies de Scarabaeidae a lo largo del año en BTC. Esto puede deberse a la 
presencia de un potrero contiguo al sitio de BTC, con la presencia de boñigas de ganado, así como a la presencia continua de excretas, cadáveres y material humificado en el bosque, a lo largo de todo el año (ver discusión de abundancia mas adelante).

También es evidente que se obtuvo una mayor riqueza en trampas de luz que en necrotrampas y coprotrampas. Esto es resultado de la naturaleza de las especies capturadas con cada método, es decir, tal vez simplemente hay más especies con fototactismo positivo que especies necrófagas y coprófagas (Morón et al. 1998, 2000; Deloya et al. 1995). Hay que mencionar que, por ejemplo, no todas las especies de Melolonthidae presentan fototactismo positivo, y por tanto es necesario establecer muestreos basados en colectas directas sistemáticas adicionales (ver Méndez- Aguilar et al. 2005). Reuniendo los datos de los tres métodos cubrimos un amplio espectro de gremios alimenticios con importancia ecológica (Morón \& Aragón 2003) y económica (Aragón et al. 2001). La riqueza total (número de especies) de ambos sitios (36 en ZA y 34 en BTC) no es diferente estadísticamente, lo cual es un reflejo de la cercanía geográfica de ambos sitios. Esta cercanía también se ve reflejada en la composición de especies y en el número de especies compartidas en ambos sitios (ver arriba).

En cuanto a la abundancia en función del tiempo (meses del año), se observa un patrón semejante al de la riqueza, pero más marcado entre las estaciones del año y entre sitios. Es probable que esta tendencia en ZA sea resultado de la mayor abundancia de recursos alimenticios en la zona agrícola (en sentido amplio), que es rica en especies cultivadas (maíz, caña, papalo, frutales, etc.) a lo largo del año, gracias a un sistema de riego que existe en el valle agrícola, alimentado por el Río Huehuetlán (ver Fig. 1). En BTC este patrón debe ser resultado del efecto de una elevada temperatura y mayor precipitación en verano, lo cual genera mayor disponibilidad de recursos alimenticios, aunque ya dijimos que no hay una correlación estadística entre abundancia y precipitación o temperatura (Cuadro 9). La abundancia por método de colecta sigue un patrón semejante al de la riqueza: una mayor abundancia en trampa de luz que en necrotrampa y coprotrampa en ZA, y lo mismo en BTC. También es mayor la abundancia en ZA que en BTC, con excepción de las coprotrampas, en donde es inversa esta tendencia. Esto mismo se ve reflejado en la mayor abundancia de Melolonthidae en ZA y la mayor abundancia de Scarabaeidae en BTC. A nivel de subfamilias (Melolonthinae y Scarabaeinae) se repite este patrón.

En total es mayor la abundancia en ZA (607) que en BTC (418) y hay una diferencia estadísticamente significativa entre estos valores. En general puede decirse que hay más recursos alimenticios para la fauna de Scarabaeoidea en ZA por la presencia del sistema de riego que funciona a lo largo de todo el año, y la fauna de BTC está a expensas de la presencia de la productividad asociada al temporal. En el bosque disminuye drásticamente la producción de biomasa vegetal en la época seca 
del año, por lo que los gremios mas destacados son los coprófagos y los necrófagos (los recursos excremento, cadáveres y material humificado puede no disminuir tanto como la materia vegetal en este período en este sitio). En cuanto a abundancia de especies, hay un mayor número de especies abundantes en ZA (cinco) que en BTC (una). Esto se verá reflejado en los índices de diversidad y de dominancia (ver mas adelante). Dicho de otra manera, hay mas especies raras en BTC que en ZA.

En cuanto a larvas, solo se puede describir la situación de la riqueza y la abundancia en cada sitio, ya que no son comparables los datos por el diferente esfuerzo de captura. En BTC y en ZA las larvas más abundantes son de Phyllophaga y los adultos más abundantes en estos sitios son de Diplotaxis y Cyclocephala respectivamente (Cuadro 6). La mayor abundancia de larvas de Phyllophaga en relación con otros géneros coincide con los resultados obtenidos por Tapia-Rojas (2005) en bosques del Izta-Popo y Teziutlán, Puebla. La mayor abundancia de larvas de Melolonthidae se observó en septiembre y octubre; esto coincide en lo general con los resultados de Villalobos (1991) en Laguna Verde, Veracruz. La abundancia de larvas por mes muestra valores altos en septiembre y octubre y valores bajos el resto del año en BTC, y en ZA la mayor abundancia se da en septiembre, con valores bajos el resto del año. Esto debe ser resultado de la fenología de las especies, por lo que habría de analizar caso por caso y tratar de criar las larvas hasta el estado adulto para identificar las especies con precisión.

\section{Diversidad y Dominancia}

La diversidad estimada a partir del Índice de Diversidad de Shannon-Wiener, es mayor en ZA (2.80) que en BTC (2.67), aunque no hay diferencia estadísticamente significativa. La equitabilidad también es mayor en ZA (0.78 vs. 0.76), pero la dominancia es mayor en BTC ( 0.11 vs. 0.09 ). De hecho, estos resultados en principio nos llevan a rechazar las hipótesis de que la diversidad es inversamente proporcional a la dominancia, y de que encontraríamos mayor diversidad en BTC y una mayor dominancia en ZA, como resultado de actividades antrópicas como la agricultura. Creemos que la diversidad es alta en ZA debido a: 1) el valle donde se encuentra la zona agrícola está rodeado de bosque, con el consecuente efecto de borde que esto conlleva (un efecto de borde es el efecto de la yuxtaposición de ambientes contrastantes en un ecosistema). Es decir, las especies del bosque, cuya área total en el municipio es mayor que la de zonas agrícolas, están influyendo mas a las faunas de ZA que a la inversa; 2) las parcelas de la zona agrícola son relativamente pequeñas (0.5-2.0 Ha) y en ellas se siembran cultivos diferentes (maíz, caña, pápalo, etc.) simultáneamente a lo largo del año. Esto da como resultado un valle agrícola "diverso" en cultivos, lo que se ve reflejado en la diversidad de fauna entomológica.

En cuanto a tipos de diversidad (en el sentido de Moreno 2001 y Halffter \& Moreno 2005) es mayor la aportación de la diversidad alfa (89.68\%) a la diversidad 
gamma, que la aportación de la diversidad beta (10.31\%). Esto es resultado de la elevada similitud (Índice de Jaccard $=48 \%$ ) entre las comunidades, por lo que la diversidad al interior de cada ambiente (diversidad alfa) tiene mas peso en la diversidad del paisaje (diversidad gamma).

\section{Especies Indicadoras}

De acuerdo con Morón (1997) y Favila \& Halffter (1997) en este análisis solo se consideraron como especies indicadoras de diversidad los Dynastinae, Rutelinae y Scarabaeinae. Al observar la abundancia relativa por especie se aprecian mas especies raras en ZA que en BTC, es decir, hay más dominancia en ZA. Esto se corrobora con el Índice de Dominancia de Simpson, que es mayor en ZA (0.17 vs 0.13 ). La riqueza (23 vs 21 ) y la diversidad (2.43 vs 2.24 ) de las especies indicadoras son mayores en BTC que en ZA, aunque no son estadísticamente significativas las diferencias. Si estimamos el Índice de Diversidad de Shannon Wiener sólo para especies indicadoras de Scarabaeinae en BTC (2.2), se asemeja al valor de 2.5 obtenido por Halffter, Favila \& Halffter (1992, in Favila \& Halffter 1997) en un bosque tropical lluvioso de Chiapas (Palenque); también la equitabilidad es semejante ( 0.81 vs 0.76 , respectivamente). Por otro lado, la abundancia de las tres subfamilias de especies indicadoras, como en el caso del análisis para todas las especies de las dos comunidades, es mayor en ZA que en BTC. Con base en estos resultados de las especies indicadoras, sólo aceptamos la hipótesis de que no hay diferencia estadísticamente significativa en los valores de riqueza y diversidad, es decir: 1) Se encontró un numero elevado de especies indicadoras en ambos sitios, equivalentes a casi el 50\% del total (24 en BTC, es decir 51\% del total, y 23 en ZA, es decir, un $48 \%$ del total). Esto sugiere por un lado que está bien conservado el bosque, y que la zona agrícola, por su cercanía con el bosque y por su manejo, presenta buenos indicadores. Para consolidar esta conclusión es necesario que se hagan estudios con otros taxones indicadores de conservación o de perturbación, como son otros coleópteros (Pearson \& Cassola 1992), otros insectos (Andersen 1997) o vertebrados (Medellín et al. 2000). 2) Se observa una mayor diversidad (no significativa estadísticamente) de especies indicadoras en BTC que en ZA. La escasa diferencia de estos valores entre ambos sitios puede ser consecuencia de que el ambiente agrícola es heterogéneo en recursos, lo cual aunado a la cercanía con el bosque, indica que es un sistema agrícola que favorece la biodiversidad de Scarabaeoidea. 3) La dominancia de especies indicadoras es mayor en ZA (0.1630) que en BTC (0.1300), pero las diferencias no son significativas estadísticamente. Este resultado y el del inciso anterior, no pueden confirmar que la diversidad es inversamente proporcional a la dominancia.

Por tanto, con base en este análisis de comunidades de Scarabaeoidea puede decirse que el efecto antrópico de las actividades agrícolas sobre el bosque es 
mínimo. Esto de acuerdo con las circunstancias de este estudio y puede depender de la fragmentación, proximidad y tamaño de las áreas antropizadas. Se pueden esperar resultados semejantes en sitios como Chamela, Jalisco porque casi no hay superficie cultivada, pero en Jojutla, Morelos los resultados podrían ser distintos porque la relación superficie cultivada/bosque es muy diferente.

AGRADECIMIENTOS. Al Dr. Agustín Aragón García por su apoyo durante los trabajos de campo y gabinete. Al Dr. Jesús Francisco López Olguín por sus acertadas observaciones a la versión preliminar del manuscrito. Al Dr. José Lino Zumaquero Ríos por su apoyo logístico durante la realización de esta investigación.

\section{LITERATURA CITADA}

Andersen, A. N. 1997. Using ants as bioindicators: multiscale issues in ant community ecology. Conservation Ecology. (1)1:8 (on line). URL: http://www.consecol.org/vol1/iss1/art8

Aragón, A. M.A. Morón, A.M. Tapia-Rojas \& R. Rojas-García. 2001. Fauna de Coleoptera Melolonthidae en el Rancho "La Joya”, Atlixco, Puebla, México. Acta Zoológica Mexicana (n.s.), 83: 143-164.

Carrillo-Ruiz, H. \& M. A. Morón. 2003. Fauna de Coleoptera Scarabaeoidea de Cuetzalan del Progreso, Puebla, México. Acta. Zoológica Mexicana (n.s.), 88: 87-121.

Deloya, C. A. Burgos, J. Blackaller \& J.M. Lobo. 1993. Los coleópteros Lamelicornios de Cuernavaca, Morelos, México. (Passalidae, Trogidae, Scarabaeidae y Melolonthidae). Boletín Sociedad Veracruzana de Zoología, 3(1):15-55.

Deloya, C. \& M.A. Morón. 1994. Coleópteros Lamelicornios del Distrito de Jojutla, Morelos, México (Melolonthidae, Scarabaeidae, Trogidae y Passalidae). Listados faunísticos de México (V). Instituto de Biología, UNAM, México. 49 pp.

Deloya, C., M. A. Morón \& J. M. Lobo. 1995. Coleoptera Lamellicornia (MacLeay, 1819) del Sur del Estado de Morelos, México. Acta. Zoológica Mexicana (n.s.), 65: 1-42.

Endrödi, S. 1966. Monographie der Dynastinae (Col. Lam.) I Teil. Entomologische Abhandlungen Museum Tierkunde,Dresden, Bd. 33: 1-457.

Favila, M.E. 2005. Diversidad alfa y beta de los escarabajos del estiércol (Scarabaeinae) en Los Tuxtlas, México. Pp. 209-219. In: G. Halffter, J. Soberón, P. Koleff \& A. Melic (Eds.). Sobre Diversidad Biológica: el significado de las diversidades Alfa, Beta y Gamma. m3m: Monografías Tercer Milenio vol. 4. S.E.A., Zaragoza.

Favila, M.E. \& G. Halffter. 1997. The use of indicator groups for measuring biodiversity as related to community structure and function. Acta Zoológica Mexicana (n.s.), 72: 1-25.

García de Jesús, S. 2006. Estudio faunístico de Coleoptera Lamellicornia de la región La Malinche, Tlaxcala, México. Tesis profesional inédita. Escuela de Biología, BUAP, Puebla, México.

Halffter, G. \& C.E. Moreno. 2005. Significado biológico de la diversidades alfa, beta y gamma. Pp. 518. In: G. Halffter, J. Soberón, P. Koleff \& A. Melic (Eds.). Sobre Diversidad Biológica: el significado de las diversidades Alfa, Beta y Gamma. m3m: Monografías Tercer Milenio vol. 4. S.E.A., Zaragoza.

INEGI. 1994. Anuario Estadístico del Estado de Puebla, INEGI. Aguascalientes, México. 618 pp.

INEGI. 2000. Síntesis Geográfica del Estado de Puebla. INEGI, Aguascalientes, México. 124 pp.

Magurran, A.E. 1988. Ecological diversity and its measurement. Princeton University Press.

Medellín, R.A., M. Equihua \& M.A. Amin. 2000. Bat diversity and abundance as indicators of disturbance in neotropical rainforests. Conservation Biology, 14(6):1666-1675. 
Méndez-Aguilar, M. J., A. E. Castro- Ramírez, R. Alvarado Barrantes, C. Pacheco-Flores \& C. Ramírez-Salinas. 2005. Eficacia de dos tipos de recolecta para registrar la diversidad de Melolóntidos nocturnos (Coleoptera: Scarabaeoidea). Acta Zoológica Mexicana (n.s.), 21(3): 109-124.

Moreno, C.E. 2001. Métodos para medir la biodiversidad. Manuales y tesis SEA, Vol.1. Zaragoza, España.

Morón, M.A. 1983. Introducción a la biosistemática y ecología de los Coleópteros Melolonthidae en México. Pp. 1-13. In: II Mesa Redonda sobre Plagas del Suelo. Sociedad Mexicana de Entomología, Colegio de Postgraduados y Universidad Autónoma de Chapingo, México.

Morón, M.A. 1990. Los coleópteros Lamelicornia de Sian Ka’an, Quintana Roo, México. Pp. 275-280. In: D. Navarro y J.G. Robinson (Eds.). Diversidad Biológica en la Reserva de la Biosfera de Sian Ka'an, Quintana Roo, México. CIQRO-PSTC-SEDUE, México.

Morón, M.A. 1994. Fauna de Coleoptera Lamellicornia en las montañas del noroeste de Hidalgo, México. Acta Zoológica Mexicana (n.s.), 63: 7-59.

Morón, M.A. 1997. Inventarios faunísticos de los Coleoptera Melolonthidae Neotropicales con potencial como biondicadores. Giornale Italiano Entomologia, 8: 265-274.

Morón, M.A. 2001. Larvas de escarabajos del suelo en México (Coleoptera: Melolonthidae). Pp. 111130. In: C. Fragoso \& P. Reyes-Castillo (Eds.). Diversidad, función y manejo de la biota edáfica en México. Acta Zoológica Mexicana (n.s.), número especial 1.

Morón, M.A. (Ed). 2003. Atlas de los escarabajos de México. Coleoptera: Lamellicornia, Volumen 2. Familias Scarabaeidae, Trogidae, Passalidae y Lucanidae. Argania editio, Barcelona.

Morón, M.A. \& A. Aragón. 2003. Importancia ecológica de las especies americanas de Coleoptera Scarabaeoidea. Dugesiana, 10(1): 13-29.

Morón, M.A. A. Aragón, A. M. Tapia-Rojas \& R. Rojas-García. 2000. Coleoptera Lamellicornia de la Sierra del Tentzo, Puebla, México. Acta Zoológica Mexicana (n.s.), 79: 77- 102.

Morón, M.A. \& C. Deloya. 1991. Los Coleópteros Lamelicornios de La Reserva de la Biosfera "La Michilía”, Durango, México. Folia Entomológica Mexicana, 81:209-283.

Morón, M.A. C. Deloya \& L. Delgado-Castillo. 1988. Fauna de Coleópteros Melolonthidae, Scarabaeidae y Trogidae de la región de Chamela, Jalisco, México. Folia Entomológica Mexicana, 77: 313-378.

Morón, M.A. C. Deloya, A. Ramírez \& S. Hernández. 1998. Fauna de los Coleópteros Lamellicornios de la región de Tepic, Nayarit, México. Acta Zoológica Mexicana (n.s.), 75:73-116.

Morón, M.A., B.C. Ratcliffe \& C. Deloya. 1997. Atlas de los escarabajos de México. Volumen 1. Familia Melolonthidae. CONABIO y Sociedad Mexicana de Entomología, A. C. México.

Morón, M.A. \& R. Terrón. 1984. Distribución actitudinal y estacional de los insectos necrófilos en la Sierra Norte de Hidalgo, México. Acta Zoológica Mexicana (n.s.), 3: 1-46

Morón, M.A. \& R. Terrón. 1988. Entomología Práctica. Publ. 22. Instituto de Ecología, A.C. México.

Pearson, D.L. \& F. Cassola. 1992. World-wide species richness patterns of Tiger Beetles (Coleoptera: Cicindellidae): Indicator taxon for biodiversity and conservation studies. Conservation Biology, 6(3): 282-292.

Pérez- García, A. 1999. Los Coleópteros Melolonthidae de la Reserva de Huautla, Morelos. Tesis Maestría en Ciencias (inédita). Facultad de Ciencias, UNAM. México.

Reyes-Novelo, E. \& M.A. Morón. 2005. Fauna de Coleoptera Melolonthidae y Passalidae de Tzucacab y Conkal, Yucatán, México. Acta Zoológica Mexicana (n.s.), 21(2): 15-49

Salamanca-Calixto, C. 2006. Fauna de Coleoptera Lamellicornia en la Región de Tlachichuca y Ahuatepec del Camino (Ciudad Serdán), Puebla, México. Tesis profesional inédita. Escuela de Biología, BUAP, Puebla, México. 
Soberón, J. \& J. Llorente. 1993. The use of species accumulation function for the prediction of species richness. Conservation Biology, 7: 139-144.

Tapia-Rojas, A.M. 2005. Estudio de las larvas de coleópteros melolóntidos edafícolas en ambientes forestales de Puebla: su posible uso como bioindicadores. Tesis de Maestría en Ciencias Ambientales (inédita). Instituto de Ciencias, BUAP, Puebla, México.

Villalobos, F.J. 1991. The community structure of soil Coleoptera (Melolonthidae) from a tropical grassland in Veracruz, Mexico. Pedobiologia, 35: 225-238. 